\title{
Dialogue sur le mariage, dialogue dans le mariage. Sur le troisième livre de la Civil Conversatione de Stefano Guazzo
}

\author{
Dialogue on Marriage, Dialogue in the Marriage. \\ On the third book of Civil Conversatione \\ by Stefano Guazzo
}

\author{
Joanna Dimke-Kamola \\ Université Adam Mickiewicz de Poznań
}

\begin{abstract}
The starting point for this study of the marriage according to Guazzo is the notion of conversation in its original meaning, as living together. The treaty of Guazzo (1574) develops the concept of « civil conversazione » in which men exchange their opinions, but especially support each other and show mutual respect and amiability, which avoids conflicts. The study shows the analogies between such ideal of communication and Guazzo's vision of the marriage - both envisaged in the humanistic and Christian perspectives: as foundations of society and means of mutual edification. Guazzo's discourse concerning conversation of the spouses oscillates between the necessity of accomodation to the spouse (related to concealment) and sincerity. Given the complexity of codes that characterize other types of communication, « marital conversazione » is presented as the closest to the ideal of transparency.
\end{abstract}

Keywords: Stefano Guazzo, Renaissance Marriage, Renaissance Family, Rules of Communication, Conversation

Quand en 1427 Bernardin de Sienne prononçait dans sa ville d'origine des sermons passionnés consacrés, entre autres, aux principes de la vie en famille, il rappelait à ses auditeurs - hommes et femmes qui se tenaient sur la Piazza del Campo séparés par un rideau - de s'abstenir de porter des habits coûteux aux coupes extravagantes. Car la richesse ostentatoire, en effaçant les différences entre les états, bouleversait l'ordre social, tandis que l'exhibition du corps pervertissait l'ordre moral, en plaçant au second plan le souci de l'âme (Bernardino da Siena, 1989 : 1068-1098). Le prédicateur formulait clairement ses reproches : ceux qui s'habillent avec démesure sont coupables puisqu'ils veulent être pris 
pour quelqu'un qu'ils ne sont pas et désirent procurer du plaisir aux autres par leur aspect extérieur ${ }^{1}$.

Cent ans plus tard - l'année de la mort de Nicolas Machiavel, deux ans avant la mort de Baldassare Castiglione - les mêmes sentences prennent en Italie un tout autre sens. Pour le cercle toujours grandissant de lecteurs, les traités sur la politique et sur les mœurs comportant des réflexions sur le caractère mensonger de l'apparence définissaient tout simplement la condition humaine dans le monde. Ces considérations, fruit des expériences amères et des observations approfondies de leurs auteurs, n'avaient pas seulement pour but d'avertir les lecteurs devant le manque de franchise chez les autres, mais contenaient un certain programme des comportements qui prenait en compte la nette séparation entre l'homme intérieur et l'homme extérieur qui ne montrait aux autres que ce qu'il voulait montrer ${ }^{2}$. Il en fut de même avec le deuxième reproche de saint Bernardin : la volonté de procurer du plaisir aux autres par son apparence, blâmable aux yeux du prédicateur, devint au $\mathrm{XVI}^{\mathrm{e}}$ siècle une exigence des bonnes manières ${ }^{3}$.

On ne veut pas dire par là qu'au $\mathrm{XVI}^{\mathrm{e}}$ siècle les prédicateurs italiens recommandaient aux fidèles de se soucier de leur apparence ou qu'aux siècles précédents les modèles mondains de la conduite liés à la culture de la cour n'existaient point. Toutefois, si autrefois l'influence de ces modèles était limitée à une élite relativement étroite, en Italie du $\mathrm{XVI}^{\mathrm{e}}$ siècle, grâce à la propagation de l'imprimerie, les œuvres analysant des mécanismes sociaux et expliquant comment se comporter sur la scène du monde parvenaient à un large cercle de lecteurs. Les traités de Machiavel et de Castiglione devinrent l'inspiration (ou au moins un point de repère) pour un nombre incalculable d'auteurs, essayant de codifier des règles de vie en société, celle-ci étant comprise comme une sorte de jeu des apparences, non plus seulement à l'usage des hommes d'État et de cour, mais aussi des personnes de différents rangs sociaux et représentant différents niveaux d'éducation.

Parmi les œuvres considerées comme fondamentales dans l'histoire de ce genre de trattatistica, la Civil conversatione de Stefano Guazzo mérite une attention particulière, non seulement en tant que texte fondateur d'un idéal de sociabilité qui va influencer fortement la formation de la culture de l'honnête homme,

\footnotetext{
${ }^{1}$ Sur les accusations formulées par Saint Bernardin et d'autres prédicateurs contre la « confusion » provoquée par les habits non appropriés, cf. Muzzarelli (2008 : 324-341).

${ }^{2}$ Pour cette problématique, voir par exemple Courtine \& Haroche (1994) et Snyder (1995).

${ }^{3}$ Voir, par exemple, les observations de Giovanni Della Casa $(1666: 30):$ « ceux qui ont des habits riches, mais mal faits font croire, ou qu'il leur est fort indifferent de plaire, ou qu'ils ne sçavent pas en quoy consiste la grace ». 〈http://www.amazon.fr/Marque-place-porte-chevaletturquoise/dp/B0092SBLZO/ref=sr_1_3 ?ie=UTF8\&qid=1435940345\&sr=8-3\&keywords=marque +place+bleu \& la propreté »> (1. ed. 1558). [<http://gallica.bnf.fr/ark:/12148/bpt6k95302j>].
} 
mais aussi en tant qu'un document intéressant sur les changements que subit le discours sur le mariage au $\mathrm{XVI}^{\mathrm{e}}$ siècle.

Publié en 1574 à Brescia, réédité la même année à Venise et jouissant en Italie et à l'étranger d'une exceptionnelle popularité pendant quelques dizaines d'années ${ }^{4}$, le traité de Guazzo est un dialogue en quatre livres, consacré à la «conversazione », entendue au sens premier de ce mot, comme «vie en commun » ou «fréquentation habituelle », et donc comme l'antonyme de « solitude ». Il faut souligner dès le début le rôle essentiel que cette œuvre jouera dans l'évolution sémantique de la notion de conversation. Basé sur une conception humaniste et chrétienne du discours comme fondement de la vie sociale et moyen de l'édification mutuelle, le traité de Guazzo - au fur et à mesure des éditions et adaptations - se transforme en un recueil de conseils et de formules utiles dans l'art de mener la conversation dans les salons ${ }^{5}$.

Guazzo montre le rôle essentiel de la «conversazione» dans la vie de l'individu et de la société. Il prouve que fréquenter les autres permet de connaître les caractères humains (y compris le sien), d'améliorer ses mœurs et d'échanger les expériences et les points de vue, ce qui favorise le développement intellectuel. Guazzo reste convaincu que la conversation forme les attitudes morales et élargit le savoir humain plus efficacement que la lecture et l'étude dans la solitude. Toutefois, pour que la «conversazione» apporte les fruits désirés, elle doit être «civile », ce qui signifie que les interlocuteurs doivent respecter certaines règles précises : indépendamment des différences qui les séparent, ils sont obligés à se témoigner respect et bienveillance ${ }^{6}$. Comme

\footnotetext{
${ }^{4}$ Durant les cinquante années qui suivirent la première édition, on publie en Italie plus de quarante éditions nouvelles. Le succès éditorial de la Civil conversatione à l'étranger est également important. Jusqu'à la fin du XVI ${ }^{\mathrm{e}}$ s. le traité est traduit deux fois en français (en 1579 paraissent deux traductions : à Lyon, celle de Gabriel Chappuys, et à Paris, celle de François de Belleforest), en anglais (1586 et 1588) et en latin. En 1598 paraissent la version allemande et, dès le début du XVII ${ }^{\mathrm{e}}$ s., les éditions en hollandais, en espagnol et en tchèque (Quondam, 1993). En France, le traité de Guazzo est largement connu et inspire de nombreux hommes de lettres (Lojacono, 2004:107, 143). Pour la Civil conversatione comme inspiration de «l'art de conférer » de Montaigne voir Boccazzi (1991 : 295-303) et Panichi (2004 : 373-390).

${ }^{5}$ Pour une analyse de la conversation inspirée de l'étymologie (versare cum, vertere cum «se tourner ensemble vers qc. ", " passer d'un état à un autre ») voir Ronchi (2003). Fumaroli $(1994: 138,327)$ remarque que le mot français «conversation » a été emprunté à la traduction française de l'œuvre de Guazzo et qu'il est entré en usage en France dans le sens d' « entretien entre gens civilisés ».

${ }^{6}$ Guazzo illustre son idée en prenant l'exemple des fabricants des cordes : «à [tous les hommes] est licite \& convenable de disputer [...], quand ils entrent en propos ensemble, \& qu'ils font de points contraires: \& est celuy digne de plus grande louange, qui defend le party plus dificile: \& combien qu'ils soient discordans en paroles, ils ne discordant neantmoins en amour, \& muluelle bienveillance, ains cherchent d'accord la verité, \& ressemblent à ceux qui font les cordes, desquels combien que l'un torde au contraire de l'autre, s'accordent neantmoins, quant à l'intention \& fin de l'œuvre » (Guazzo, 1580 : 99 ; Guazzo, 1993 : 63-64).
} 
explique l'auteur de la première traduction française, Gabriel Chappuys, en paraphrasant la lettre dédicacée jointe à l'édition italienne du traité, Guazzo a écrit son œuvre en considérant :

[...] que les debats $\&$ scandales qui pour la plus part écheent entre les hommes, ne viennent d'autre chose, que de ne pouvoir se servir des manieres propres \& convenables en la conversation, \& qu'à cete raison, s'il pouvoit tant faire que de monstrer à chacun ce que, selon son estat, il luy faut faire, pour hanter \& converser avec les autres, il feroit chose merveilleusement profitable au monde [...] (Guazzo, 1580 : fragment non paginé ; Guazzo, $1993: 1$ ).

Le but que se fixe Stefano Guazzo en composant la Civil conversatione est de prévenir les conflits et les scandales. Le moyen qui doit assurer aux hommes une vie harmonieuse dans la communauté est l'acquisition par chacun d'un certain répertoire de comportements adéquats, correspondant à sa condition sociale, et ensuite leur adaptation à la situation dans laquelle se déroule la conversation. Ce qui est important, c'est également le fait que le principe d'adaptation-comme c'était dit dans Le livre du courtisan - prend en compte le niveau intellectuel et même les opinions des interlocuteurs. Un homme bien élevé ne manifestera pas son avantage dans la conversation, mais il cachera plutôt son érudition. Il n'essaiera pas de combattre ses interlocuteurs, mais au contraire, il tentera d'obtenir un consensus ou un compromis. Dans le cas des conversations avec les dames, cette règle prend même la forme de l'ordre de plier ses opinions aux leurs.

Pour éclairer sa réflexion, Guazzo traite l'une après l'autre les différentes situations de communication et les différentes catégories d'interlocuteurs. Ainsi, le premier livre qui est un éloge général de la conversation, est suivi du second, consacré à la "conversazione publica», et du troisième, dont le sujet est la «conversazione privata» appelée également «famigliare» ou "domestica». En ce qui concerne la classification des interlocuteurs, l'auteur adopte les critères suivants : l'état social, l'âge, le sexe, le statut économique, la profession, mais aussi les traits de caractère, décrits avec une grande précision psychologique et divisés en catégories et sous-catégories.

Aussi bien dans les indications concernant le comportement dans l'espace public que dans les conseils se rapportant aux relations entre les habitants de la maison, l'accent est mis sur le respect de la hiérarchie définie comme naturelle. En plus, ceux qui sont de rang plus élevé (les nobles, les riches, les pères de famille) sont incités à montrer de la douceur dans les contacts avec leurs subordonnés, car, selon l'auteur, c'est seulement de cette manière qu'outre le respect («onore ») de leurs interlocuteurs, ils peuvent obtenir aussi leur bienveillance («benivolenza ») qui, d'après Guazzo, est le plus important élément de cohésion de la communauté (« il vero legame della conversazione ») (Guazzo, 1993 : 91). 
Cette courte présentation des principes du traité est nécessaire pour connaître le contexte dans lequel se situent les réflexions de Guazzo au sujet du mariage et pour saisir l'idée de la "marital conversazione». Les considérations sur le mariage occupent une grande partie du livre consacré à la «domestica conversazione » et précèdent les remarques sur les relations entre les parents et les enfants, entre les frères et les sœurs ainsi qu'entre les maîtres et les domestiques. La problématique de la gestion des relations interfamiliales est un élément permanent des livres de ménage, populaires dans la culture ancienne. Le deuxième sujet traditionnel de ce type de livres représentant le genre remontant à l'Antiquité : oeconomica, est l'administration des biens de la famille. Guazzo s'éloigne toutefois de la convention des oeconomica : il renonce à ce sujet, en expliquant que la première condition de l'harmonie de la maison ne repose pas sur les affaires matérielles, mais sur les bonnes manières et sur la communication réussie entre les personnes vivant sous le même toit.

Pourtant, dans la suite de ses réflexions sur la famille, l'auteur de la Civil conversatione se conformera plutôt strictement aux schémas de composition et d'argumentation traditionnels des traités sur le mariage. Il commence donc par la question "Cela vaut-il la peine de se marier? », à laquelle il répond assez rapidement en faveur du mariage. À l'époque où il écrit - en dehors de la convention comique et de celle du "paradoxe humaniste»-il est rare que les œuvres déconseillent de fonder une famille. La riche topique misogame (et misogyne) est utilisée essentiellement dans les jeux de société et dans les exercices d'éloquence appartenant au genre suasio et dissuasio nubendi. Comme le dialogue de Guazzo se présente sous la forme d'un tel jeu conversationnel, mené dans la bibliothèque de la maison par deux amis, le chevalier Guillaume Guazzo et le docteur Annibal Magnocavalli, le problème de l'utilité du mariage est réparti sur deux voix. L'échange des avis n'a cependant pas d'aspect de polémique oratoire : en initiant la conversation, les interlocuteurs se sont mis d'accord que, pour éviter la «pomposa dottrina», dans la discussion ils feront référence plutôt à leur propre expérience, à leur bon jugement et à l'opinion commune. "La commune opinione » signifie chez Guazzo également la sagesse populaire, et ce que l'on peut nommer l'autorité collective des classiques de la pensée. Dans la discussion sur le mariage, on ne cite pas les noms des savants, mais seulement des sentences et des anecdotes. Il ne s'agit pas de combattre son interlocuteur ni même de le convaincre mais de passer le temps de façon agréable et à la fois utile, car la réflexion commune sur le mariage doit aider à comprendre comment éviter les problèmes conjugaux.

Comment les éviter? Rappelons le moment où les deux interlocuteurs se mettent d'accord sur le fait qu'il existe d'autres méthodes moins radicales pour éviter les problèmes matrimoniaux que de persister dans le célibat. Dans cette partie du dialogue domine une vision du mariage associée principalement à la 
charge des devoirs et au risque d'échec, même si elle n'est pas dépourvue d'éléments positifs, tels les enfants, présentés ici, conformément à la tradition antique, comme une forme terrestre de l'éternité. Dans l'argumentation promatrimoniale du Seigneur Annibal, on perçoit, à peine audible, un écho de l'idéal humaniste de la vie active, avec une louange de la famille comme le fondement du bien-être de l'individu et de l'État. Dans cette partie de la discussion on trouve très peu de renvois au Concile de Trente qui, à peine quelques années avant la composition de la Civil conversatione, confirma solennellement la dignité sacramentelle de l'état du mariage. Chez Guazzo, cette apologie du mariage est absente. Les influences des idées humanistes et post-tridentines en matière de la vie familiale sont visibles chez lui plutôt dans le postulat du choix attentif de la compagne de la vie. Rappelons que déjà au $\mathrm{XV}^{\mathrm{e}}$ siècle, les apologistes du mariage reconsidèrent les attitudes misogynes en prouvant que la raison des malentendus entre les conjoints n'est pas la nature perverse des femmes - êtres faibles et imparfaits dont une certaine partie des hommes doit supporter la pénible compagnie pour assurer la pérennité de l'espèce humaine - mais l'incompatibilité des prédispositions ou le manque de préparation à l'accomplissement des rôles de mari et de femme. Le choix du conjoint adéquat est devenu primordial, tout comme un programme d'éducation visant à former chez l'homme, dès son enfance, des traits de caractère et des capacités garantissant le bonheur familial (Fubini Leuzzi, 1996 : 253-267). Inculquer aux jeunes gens des principes qui pussent les guider dans le choix du conjoint ainsi que les préparer aux rôles qui les attendaient dans la vie adulte était également une partie importante de l'idéologie profamiliale du Concile de Trente.

Quand le Seigneur Annibal dans la Civil conversatione donne à son plus jeune interlocuteur des conseils concernant les critères du choix de l'épouse, ce sont, dans leur majorité, des lieux communs du discours contemporain sur le mariage (Patrizi, 1990 : 73sq. ; Frigo, 1990 : 121-145). Le Chevalier Guillaume apprend donc (à supposer qu'il l'ignorât) que les plus grands obstacles à l'harmonie familiale sont : la différence importante de l'âge ou du statut social des conjoints, le mariage contre la volonté de l'une des parties ainsi que les problèmes liés au montant de la dot. Ce ne sont pas les recettes d'un mariage réussi qui distinguent le traité de Guazzo des autres, mais plutôt sa manière d'argumenter mettant en valeur l'importance des apparences et soulignant le changement des mœurs. Observons de près la question de l'âge des époux. Pour convaincre que les maris ne doivent pas être beaucoup plus âgés que leurs femmes, Guazzo met l'accent sur le malheur des épouses qui, même si elles sont le plus fidèles, ne peuvent pas éviter une mauvaise réputation, car, pour l'opinion publique, la barbe blanche de l'époux est la preuve suffisante de l'adultère féminin (Guazzo, 1993: 180). Une autre remarque de Guazzo portant sur l'âge des époux est en même temps une réflexion sur la mutabilité des mœurs. Il s'agit de 
l'ancienne recommandation d'Aristote, selon qui les hommes et les femmes doivent se marier respectivement à l'âge de trente-six et dix-huit ans - une recommandation que "per necessità dei tempi» personne n'observe plus (Guazzo, 1993 : 78).

Outre les questions concernant l'âge, le statut social ou la dot, un point incontournable des débats sur le choix de l'épouse était sa beauté. Le fragment du dialogue de Guazzo relatif à ce problème prend la forme d'un recueil de proverbes et de topoï, plutôt que d'un discours cohérent. Les avertissements de l'Ancien Testament devant une mulier pulchra et fatua ou superba y côtoient une anecdote au sujet de l'épouse laide qui malgré tout est chère au cœur de son mari, cela veut dire qu'il la traite comme toutes autres choses précieuses: il l'économise et, comme Guillaume le commente malicieusement, «il ne s'enivre pas de son vin» (Guazzo, 1993 : 183). Comme d'autres écrivains de son siècle, Guazzo résout le dilemme entre le choix de la beauté dangereuse et la laide quiétude, en recourant à la règle du juste milieu :

Il y a long temps, que i'ay apris que la prefection du corps consiste en la mediocrité, de sorte qu'il ne soit ny trop robuste, ou beau, ny trop foible ou diforme, pource que l'un rend les personnes audacieuses \& fieres ; l'autre, abiectes \& pusilanimes. Et pourtant est grandement exaltee la moyenne beauté, qui est propre à la femme \& blasmee l'extremité de la beauté \& de laiddeur : pource que l'une tourmente, \& l'autre foule (Guazzo, 1580 : 295 ; Guazzo, 1993 : 184).

Dans ce fragment, le rapport entre ce qui est à l'extérieur et ce qui est caché dans les profondeurs de l'âme/cœur/esprit, revient comme un leitmotiv. La beauté exceptionnelle est envisagée comme une image corporelle des traits du caractère tels que la hardiesse et l'insolence, par contre la laideur devient un avertissement devant la pusillanimité et la lâcheté. Le fragment du dialogue consacré à des interventions cosmétiques est une mine de jugements sur le rapport entre le caractère de l'homme et l'aspect extérieur. Guazzo puise à pleines mains dans la tradition des siècles écoulés :

A la verité, ie ne fay bon iugement de telles femmes, \& estime que ny plus ny moins qu'elles ont les couleurs de leurs visages faintes, elles portent aussy un cœur faint, de maniere que l'on ne peut attendre d'elles une pure \& simple affection (Guazzo, 1580 : 298 ; Guazzo, 1993 : 185).

Dans la suite du dialogue, quand les interlocuteurs commencent à esquisser l'image d'une épouse idéale, ils ne manqueront pas d'apprécier « un maquillage sincère » («virtuoso belletto »), c'est-à-dire des joues rosies par les travaux ménagers (Guazzo, 1993: 207). La rougeur vigoureuse est un signe pour l'observateur perspicace, envoyé par la nature même. Le rouge à joues fausse 
cette communication naturelle. Dans un autre fragment du dialogue, la femme qui se maquille est considérée comme une falsificatrice qui change la création de Dieu. Si cette pensée est fortement présente dans le discours traditionnel sur le mariage, au moins à partir de Tertullien, une longue série de remarques que Guazzo consacre à la « signification » des gestes, des vêtements et des coiffures semble prendre sa source dans l'observation de la vie sociale.

Le sujet des gestes et des ornements peu pudiques qui témoignent de la vanité est constamment exposé dans les anciens traités. La tête penchée, l'économie dans les gestes et les paroles, un habit modeste, les rares sorties de la maison, composent l'image idéale de la femme que Carla Casagrande a appelée, de façon pertinente, «femme gardée » (Casagrande, 1991; Majorana, 1996). La réduction des contacts avec l'extérieur est liée aux restrictions relatives aux soins du corps et de l'apparence, visant à mieux soigner la beauté intérieure ${ }^{7}$. En ce qui concerne ce sujet, Guazzo ne s'écarte pas de la rhétorique traditionnelle et met le signe d'égalité entre « corps pompeux » et « esprit vain » (Guazzo, 1580 : 330 ; Guazzo, 1993 : 203). Beaucoup plus intéressants sont des exemples concrets à l'aide desquels il illustre ses opinions sur les mœurs des femmes. La coiffure en forme du cœur percé d'une flèche, ornée d'un plumet ondoyant, est, selon Guazzo, la meilleure preuve de légèreté et d'inconstance («leggierezza e instabilità del cervello »). En appelant cette coiffure excentrique «drapeau de la vanité », Guazzo fait allusion à une coutume très populaire à son époque qui consistait à orner des vêtements des emblèmes et des devises dans le but de communiquer les intentions des personnes qui les portaient. Chez Guazzo, la coiffure précieuse est considérée comme un élément du message emblématique. Et comme l'emblème est composé d'une image (dite « corps ») et d'un motto ou titre (dit «âme» de l'emblème), Annibal cherche à déchiffrer le sens de la chevelure féminine en complétant l'image par un titre qui, selon lui, doit être suivant : « Offense à Dieu, esperance aux amoureux, ruine aux maris » (Guazzo, $1580: 326$; Guazzo, $1993: 201)$.

La «conversazione» rappelle donc un jeu consistant à déchiffrer un code emblématique: en analysant attentivement les tenues portées par différentes personnes, tout comme leurs gestes et coiffures, l'observateur recueille les données qui lui permettent de deviner l' « anima» à partir du «corpo». Il est d'ailleurs symptomatique que, sur les pages de la Civil conversatione, les conse

\footnotetext{
${ }^{7}$ « Toute prescription adressée aux femmes - la mesure dans les aliments, la modestie dans le geste, l'usage modéré de la parole, l'abandon du fard et des parures, la restriction des allées et venues, l'accès limité au monde de la culture et du travail - s'inscrit dans un double processus de dévalorisation du dehors et de valorisation du dedans. D'un côté, la femme est écartée de la vie publique et extérieure de la communauté, confinée dans l'espace privé et intérieur des maisons et des monastères; d'autre part, elle est amputée de l'extériorité de son corps et assignée à l'intériorité de son âme » (Casagrande, 1991 : 105).
} 
ils qui ont pour rôle de faciliter une communication efficace avec les dames ne portent presque jamais sur la conversation entendue au sens d'un échange de mots $^{8}$. C'est un problème intéressant qui mériterait une étude approfondie. Limitons-nous à n'en signaler que certains aspects particulièrement dignes d'attention.

Comme on l'a vu au travers des exemples analysés, dans la plupart des cas, la « lecture » des messages de nature corporelle amène les personnages de Guazzo à tirer des conclusions peu favorables à l'égard de leurs interlocutrices. Le plus souvent, les «accoustrements affectez» («affettati ornamenti ») révèlent la malhonnêteté de celles qui les portent ou tout au moins mettent en doute leur honnêteté, ce qui revient d'ailleurs au même dans une société qui attachait une si grande importance à la réputation et dans laquelle l'opinion publique représentait un puissant facteur normatif dans le domaine des comportements sociaux (Guazzo, 1580 : 329 ; Guazzo, 1993 : 203). C'est justement cette relation peu claire entre les coutumes (vertueuses ou non) d'un côté et l'opinion publique de l'autre, qui semble intriguer d'une façon plus particulière les interlocuteurs de Guazzo. Les auteurs des anciens textes à vocation pédagogique lançaient souvent aux femmes l'avertissement qu'elles devaient protéger contre toute souillure non seulement leur conscience, mais aussi leur bonne réputation. Guazzo s'inscrit dans cette tradition, mais dans ses réflexions au sujet de l'honnêteté authentique ou apparente il est possible de retrouver des influences du débat sur la simulation et la dissimulation, mené au $\mathrm{XVI}^{\mathrm{e}}$ siècle. Dans son traité, l'auteur consacre beaucoup de place à la description du chaos introduit dans le domaine de la communication sociale par les femmes qui, selon Guazzo, s'habillent de façon inadéquate par rapport à leur «statut moral ». "Vostre soeur semble chaste, en sa chaste parure, / Bien que de chasteté l'honneur ne puisse avoir. / De vous nommer putain, on vous feroit iniure / Mais pour une putain l'ornement vous fait voir » (Guazzo, $1580: 329$; Guazzo, 1993 : 203) - Annibale, qui cite ce quatrain contemporain, remarque que même le droit civil ne prévoit aucune peine à l'égard de celui qui tenterait de séduire « une honeste matrone impudiquement vêtue »(Guazzo, 1993 : 203, phrase omise dans la traduction).

L'énorme importance de l'opinion publique ressort aussi au travers de ce raisonnement curieux du Chevalier Guillaume :

Ie pense que comme ie ne suis pas content en ma conscience d'estre homme de bien, mais ie desire que le monde le sache, \& en soit certain par experience, aussy les femmes de cete ambition veulent estre courtisées \& espruovees à fin que par leurs honnestes responces elles soient mises au catalogue de femmes de bien (Guazzo, 1580 : 323 ; Guazzo, 1993 : 200).

\footnotetext{
${ }^{8}$ Pour la problématique du « silence des femmes » cf. les remarques de Zancan (1983: 13-56) consacrées aux attitudes des devisantes dans Le livre du courtisan et Furlan (2004 : 18).
} 
Ainsi, en cherchant la raison pour laquelle les femmes se pomponnent au risque d'une atteinte à leur réputation, Guillaume évoque les motifs qui sont à l'origine de son propre comportement. Il parvient à la conclusion que ce qui pousse les femmes modestes à se comporter d'une manière immodeste devant les autres c'est justement leur désir de modestie. En coquetant, les femmes se procurent une occasion de refuser à leurs adorateurs toute faveur autre qu'un regard ou un sourire bienveillant, ce qui donne à tous la preuve de leur honnêteté. Autrement dit, tous les efforts effectués par les femmes pour faire reconnaître leur beauté ne sont qu'un moyen de faire voir leur vertu.

Face à un système des codes de communication aussi complexe, le jeune homme choisissant une candidate censée devenir sa future femme se trouve devant une tâche qui n'est pas facile. Ainsi, après avoir présenté toute une série de pièges liés au choix de la candidate reposant sur une analyse purement visuelle, Annibal conseille au Chevalier Guillaume de chercher plutôt «à l'oreille». Pourtant, il ne s'agit nullement de se mettre à l'écoute des femmes, mais de prêter l'oreille à la voix de l'opinion publique. Annibal recommande de « adviser sur tout aux qualitez de la femme, \& considerer la vie du pere \& de la mere » (Guazzo, $1580: 300$; Guazzo, $1993: 187)$ et il déplore que les coutumes qui règnent en Italie, à la différence de celles qui règnent en France, ne permettent pas de visiter les maisons des jeunes demoiselles qu'on destine au mariage ni de converser librement avec leurs habitants. La possibilité de connaître la famille de la candidate devrait fournir aussi bien des informations sur la nature de celle-ci (c'est-à-dire sur les habitudes héritées de ses parents) ainsi que sur sa culture (c'est-à-dire sur les qualités acquises grâce à l'éducation). $\mathrm{Ce}$ thème est constamment présent dans les traités sur le mariage. Néanmoins, ce qui, parmi les préceptes relatifs à l'éducation d'une jeune fille, devrait attirer notre attention, c'est la réflexion de Guazzo à propos des différences culturelles entre les régions. Cette observation lui permet de formuler la thèse selon laquelle il serait opportun d'éduquer les jeunes filles conformément aux règles de la vie en société qui sont en vigueur dans le pays de leur futur mari. Pour être plus convaincant, Guazzo évoque l'exemple des Romains qui cachent leurs femmes devant les autres, et celui des Siennois dont les femmes participent à la vie de la société. Ainsi, le fait d'adapter le modèle éducatif aux coutumes d'une autre ville devrait permettre aux jeunes femmes d'éviter la mauvaise opinion de leurs futurs concitoyens. Dans le cas contraire, une jeune fille originaire de Rome pourrait passer à Sienne pour maladroite, voire grossière, sans parler du risque d'avoir une mauvaise réputation qu'encourait une jeune Siennoise dans la Ville éternelle (Guazzo, 1993 : 190).

Dans les réflexions concernant la préparation aux rôles d'époux et d'épouse, on souligne la nécessité de s'adapter aux autres, principe clé de la «civil conversatione ». Selon Guazzo, de la même manière que l'on devrait chercher à suivre 
le fil de la discussion, il faudrait adapter les coutumes de la future épouse aux formes de relations sociales acceptées dans le milieu du futur époux. Guazzo développe ici les conceptions de la vie en société répandues par Le livre du courtisan et par le Galatée, basées sur l'éthique du juste milieu d'Aristote et sur le decorum de Cicéron. Rappelons ici que, dans l'interprétation de Della Casa, l'idéal de mediocritas et de decorum avait pris la forme d'une recommandation bien précise : "On ne doit donc point s'opposer aux coûtumes publiques, au contraire, il les faut suivre en gardant tousiours une certaine mediocrité », ou, en termes encore plus concrets, celle de s'habiller à la manière des Vénitiens lorsqu'on arrivait à Venise ou encore de ne pas porter des bérets allemands en Italie (Della Casa, 1666 : 29) (« si dee l'uomo sforzare di ritrarsi [...] al costume degli altri cittadini, e lasciarsi volgere alle usanze ». Della Casa, $1994: 18$ ).

Il convient de remarquer ici le double sens du terme italien « costume » qui d'un côté signifie une forme de comportement (coutume) et de l'autre côté - un vêtement ${ }^{9}$. De la même manière qu'un vêtement donne la forme au corps humain, les coutumes forment (con-forment) les penchants naturels de l'individu, en les adaptant aux normes acceptées dans une société donnée. Dans les traités des bonnes coutumes datant de la Renaissance nous remarquerons un contraste très fort entre le corps naturel et le corps socialement formé. Il ne convient pas de montrer publiquement le corps naturel, les autres ne devraient pas sentir son odeur ni même entendre prononcer le nom de celui-ci (le Galatée suggère de remplacer les mots «corpo» et «ventre» par le mot «pancia», jugé plus décent) (Vanni, 2006 : 383-390). Il en est tout autrement avec le corps vêtu. Couvert de beaux habits, embaumé de parfums, modélé par les gestes, il sert à être montré, à attirer l'estime des interlocuteurs, à assurer les faveurs de la société ou celles d'un prince. «Il corpo costumato ${ }^{10}$ c'est tout ce que l'homme laisse voir à l'extérieur, y compris les émotions, les sentiments et les pensées dans la mesure des convenances. Le lieu des passions et des penchants dans leur forme naturelle est le cœur (l'âme, l'esprit), c'est-à-dire l'intérieur de l'homme $^{11}$. Dans la «civil conversatione » l'homme naturel est représenté avec grâce et discrétion par son ambassadeur - l'homme extérieur.

${ }^{9}$ Aussi bien la première que la deuxième acception du terme sont des indicateurs d'appartenance de l'individu à un groupe social donné. Pour cette problématique envisagée dans la perspective anthropologique voir Remotti (1993 : 34sq.) qui analyse les liens sémantiques habitus / abito, mores / moda etc.

${ }^{10}$ L'expression vient de Laura Vanni qui a défini comme « costumato » un corps « che si anima, che si rappresenta, che si mostra, che si fa leggibile, esternando tutta la sua potenzialità comunicativa » (Vanni, $2006: 386$ ).

${ }^{11}$ Voir à ce sujet le chapitre «Questioni di stile: mediocrità e bon ton passionale », in Magli, 1995 : 239-247. 
Quel est le rapport de ces conceptions avec la vision du mariage ? La «marital conversazione », c'est-à-dire la conversation entre les époux, est-elle libre de ces convenances? Lorsqu'un jeune homme, instruit par les enseignements d'Annibal, aura choisi pour épouse une femme qui ne recourt pas au rouge à lèvres pour falsifier son visage et qui ne coquette pas, même dans le but de démontrer sa propre vertu, est-ce que la maison deviendra alors un espace libre de toute dissimulation? Ce serait tout à fait indésirable, telle est la réponse que semble donner Guazzo. S'il est vrai que dans la "marital conversazione », l'élément principal qui assure la liaison est la "benevolenza» et les époux devraient avant tout s'aimer et partager leurs soucis et leurs joies, il est tout aussi vrai que dans la conversation entre le mari et la femme, comme dans toute autre " conversazione civile », le principe auquel on doit impérativement se soumettre est celui de s'adapter constamment à son interlocuteur. De manière analogue à celle qu'on observe dans les relations qui ont été classifiées par Guazzo - et bien avant lui par Aristote (2014: 180) - comme des relations hiérarchiques, c'est avant tout la partie inférieure - dans ce cas : la femme - qui devrait chercher à s'adapter à l'homme. C'est sa « seconde nature » qui doit être formée dans la maison familiale suivant les coutumes du futur mari, alors que son visage, tel un miroir, doit refléter les tristesses et les joies de l'époux :

comme le mirouer qui represente triste le visage d'un qui est ioyeux, ou au contraire, ioyeux \& gay le visage de celuy qui est triste, ne vaut rien, ainsi est folle la femme, laquelle voyant son mary ioyeux, se fache, \& le voyant faché, se reiouit. Et pourtant qu'elle soit resoluë à se conformer aux pensees de son mary, \& à iuger les choses douces \& ameres à l'appetit de son mary, pource que la diversité des mœurs n'est aucunement propre à la conservation de l'amour [...] (Guazzo, 1580 : 331 ; Guazzo, 1993 : 204).

L'analogie proposée par Guazzo (et avant lui par Plutarque et par Érasme) entre l'époux et l'épouse et entre le visage et son reflet dans un miroir, en rappelle une autre qui était très populaire à l'époque: celle qui représentait le couple harmonieux à l'image du soleil et de la lune. Pourtant, si cette dernière image exprime principalement la soumission de la femme au mari ${ }^{12}$, la métaphore du miroir, qui renvoie à la problématique de l'expression des émotions, voire de l'empathie, ouvre une perspective particulièrement intéressante dans le discours

\footnotetext{
${ }^{12}$ Voir, par exemple, Dialogo della istitutione delle donne, un des plus populaires textes sur le mariage en Italie du XVI ${ }^{\mathrm{e}}$ s., dont l'auteur, Lodovico Dolce, menace les femmes insubordonnées d'une vision du chaos causé par la Lune qui «non si contentando della bellezza del suo cielo », avait décidé de prendre la place du Soleil. Plus loin nous avons une remarque qui met en évidence la différence entre les deux visions du mariage : chez Guazzo - empreinte avant tout d'amour, chez Dolce - de soumission et d'obéissance : « [La moglie] dee veramente istimare, che i costumi del marito le siano legge della sua vita: la qual legge l'è imposta da Dio per il legame e congiungimento del matrimonio » (Dolce, 1545).
} 
sur le mariage. Guazzo rend les maris responsables des comportements de leurs femmes, mais non pas de la même façon dont le mari - éducateur sévère était, selon de nombreux auteurs anciens, responsable du comportement d'une élève difficile à subjuguer (Vecchio, 1991: 135sq.). Chez Guazzo, le visage de la femme doit refléter la tendresse, le respect et la confiance visibles sur le visage de son mari. Suivons la conversation d'Annibal et de Guillaume à propos de la confiance :

Le Chev.[aliere] : [ie] croy que peu se trouvent au monde, encores qu-ils fassent bonne mine, qui se fient en leurs femmes.

Annib.[ale] : Ie vous en croy : mais me sçauriez vous dire, d'où vient cete commune deffiance?

Le Chev. : Paravanture de la fragilité \& foiblesse de la chair que l'on attribue volontiers à plusieurs femmes.

An. : Mais plustost de la debilité d'amour qui se doit atribuer à plusieurs hommes. Faites vostre compte, que par la porte où entre le soupçon, sort l'amour : \& si d'avanture se presente au mary quelque occasion de deffiance, qu'il examine bien sa vie, \& et il trouvera que l'occasion vient de luy, \& qu'il ne l'a parfaitement aymee : \& s'il s'en repent, \& qu'il cómance une fois à la regarder, comme la moitié de soy mesme, $\&$ à l'aimer de bon cœur, il commancera aussy à bannir le soupçon, \& s'appercevra que qui ayme est aymé [...] (Guazzo, $1580: 311$; Guazzo, $1993: 194)$.

Dans ce court dialogue, deux visions du mariage se distinguent. Guillaume représente le point de vue traditionnel sur la faiblesse morale des «filles d'Ève ", comparables, en ce qui concerne leur inclination au péché, aux jeunes enfants qui exigent un contrôle constant et des actions disciplinaires ${ }^{13}$. Ce qui frappe, c'est que le comportement des maris dont parle Guazzo est assujetti à l'opinion commune: ils se laissent guider par ce que «si suole attribuire a molte donne» («l'on attribue volontiers à plusieurs femmes») (Guazzo, 1993 : 194). La réplique d'Annibal déjà par sa forme (« si dee attribuire a molti uomini »- «se doit atribuer à plusieurs hommes ») s'écarte de la rhétorique misogyne : au lieu de suivre les stéréotypes, le docteur Magnocavalli propose de regarder de plus près sa propre vie.

L'insistance sur la responsabilité du mari et sur la réciprocité des sentiments rapproche la vision du mariage proposée par Annibal de l'image de l'amour conjugal présentée dans I libri della famiglia de Leon Battista Alberti, datant de la première moitié du $\mathrm{XV}^{\mathrm{e}}$ siècle $^{14}$. Remarquons que l'apologie du mariage

${ }^{13} \mathrm{Au}$ sujet de la formation de l'image de la femme en tant qu'être imparfait et immature cf. De Matteis (1982) et Duby (1981 : 28-33).

${ }^{14}$ Comme le montre Anna Loba (2010), des traces d'une pensée moderne sur le mariage conçu comme «alliance » fondée sur l'amour des époux, et sur le rôle de l'épouse - conseillère, aide et «compaigne» de son mari, sont déjà présentes, au seuil du $\mathrm{XV}^{\mathrm{e}}$ s., dans la pensée de 
s'accompagne dans ces textes d'un éloge du discours qui permet aux hommes d'échanger leurs pensées et leurs sentiments. Chez Guazzo - comme chez Alberti (1994 : 46) - aussi bien la parole que le mariage sont envisagés comme fondements de la vie sociale et comme moyens de l'édification mutuelle.

[...] la mesme nature a donné la parolle à l'homme, non pas à fin qu'il parle en soy mesme, qui seroit en vain, mais à fin qu'il s'en serve avec les autres: $\&$ vous voyez que la langue nous sert à enseigner, demander, conferer, negocier, conseiller, corriger, disputer, iuger, \& à exprimer l'affection de nostre cœur, moyens par lesquels les hommes viennent à s'aymer \& conioindre ensemble [...] (Guazzo, 1580: 30-31; Guazzo, $1993: 27)$.

[...] il faut qu'il [le mary] tienne la femme quasi pour un sien thresore en terre, $[\ldots]$ se souvenant que sur toute autre chose, le mary doit à sa femme fidele \& saincte compagnie $\&$ pour cette cause qu'il mette toute peine de la tenir chere $\&$ sans tache, $\&$ ne dedaigne aussy, en signe d'amitié, de luy communiquer ses pensees, car plusieurs se sont, à leur profit, conseillez avec leur femmes. Et l'homme est heureux, lequel, communiquant ses travaux à sa chere \& fidele compagne, en reçoit bonnes responces \& amiables conseils : il est heureux de ce que luy participant sa bonne fortune, il sent par la vraye allegresse de la femme redoubler la sienne (Guazzo, $1580: 315$; Guazzo, $1993: 196)^{15}$.

Guazzo souligne la sincérité qui caractérise la « conversazione » entre les époux et qui cimente leur union définie ici comme amitié («ne dedaigne aussy, en signe d'amitié, de luy communiquer ses pensees $»)^{16}$. On retrouve le même idéal de mariage dans les colloques «matrimoniaux»d'Érasme $(1523)^{17}$. Lui aussi met en évidence le rôle de la parole et du partage des sentiments dans le

Christine de Pizan, dont Le Livre des Trois Vertus révèle une proximité avec les idées proférées plus tard par Érasme.

${ }^{15}$ Cf. Alberti (1994 : 93) « se la benivolenza cresce per conversazione, con niuna persona manterrai più perpetua familiarità che colla moglie ; se l'amore si collega e unisce discoprendo e comunicando le tue affezioni e volontà, da niuno arai più aperta e piana via a conoscere tutto e dimonstrarti che alla propria tua donna e continua compagna ».

${ }^{16} \mathrm{Il}$ est intéressant de voir comment la même question est abordé par Dolce (1545). Après avoir fait l'éloge des «douces conversations » entre l'époux et l'épouse, Dolce souligne la nécessité d'une sincérité absolue de la femme, en permettant au mari de tenir secrètes ses pensées devant elle (« come niuna parte del corpo della moglie è segreta al marito: così medesimamente niun suo pensiero, niun suo desiderio gli dee essere ascoso: e questi, sì come strale a giusta meta, debbono terminare nel voler suo. All'incontro, se'l marito ripone nel suo petto alcuna cosa, la quale egli non vuole, che venga a notitia della moglie; essa si dee acquetare, e non ricercar di saperla $»)$.

${ }^{17}$ Si magnam quandam animi delectationem ex reliquorum necessariorum benevolentia capimus, quam dulce imprimis sit habere qui cum animi tui secretos affectus communices, qui cum perinde ut tecum loquaris, cuius fidei te tuto committas, qui tuas fortunas esse ducat, quid tu credis habere felicitatis mariti uxorisque coniunctionem, qua nulla possit in rerum natura inveniri, neque maior neque firmior? Cité d'après Leushuis (2003: 72). 
développement de l'amitié entre mari et femme ${ }^{18}$. Comme le remarque Reinier Leushuis, «l'union des conjoints unis dans le mariage (con-iugium) se fonde particulièrement sur l'échange de la parole entre les époux en tant qu'interlocuteurs $(\operatorname{co}(n)$ - loquium)» (Leushuis, 2003 : 74). Pour mieux comprendre l'idée de la «marital conversazione » chez Guazzo, on peut également évoquer la thèse, formulée par Leushuis dans ses études sur Érasme, concernant l'analogie entre l'idéal du dialogue humaniste et l'agape chrétienne. Sa validité semble confirmée par l'œuvre de Guazzo. Lorsque Guillaume et Annibal traitent des modalités de la conversation "civile », ils indiquent, comme exemple et modèle à imiter, l'esprit amical qui règne au cours des banquets en France :

Le Chev.[aliere]: Ie pense qu'entre plusieurs passetemps \& plaisirs, par le moyen desquels les esprits sont recrees, tiennent le premier lieu les festins non somptueux, mais familiers, proposez par un poëte pour la felicité de la vie humaine.

An.[nibale] : Comme les banquets solennels sont rempliz de bruit \& de confusion, ainsi les particuliers sont plains d'amitié \& de repos : \& comme ceux là par la diversité $\&$ delicatesse des viandes invitent les personnes au plaisir \& repletion du corps, ainsi les particuliers, avec la sobrieté les invitent à la consolation de l'esprit.

Le Chev. : Ie ne me puis tenir de louer souvent la treshumaine coustume de France, ou les parens, les amis \& les voisins s'accordent à porter chacun sa portion ordinaire, ores en la maison de l'un, ores de l'autre, où [...] laissans hors la porte toutes leurs ennuyeuses pensées, souppent ioyeusement en merveilleuse amitié \& concorde (con una maravigliosa carità e concordia) (Guazzo, 1580: 278-279; Guazzo, 1993 : 175)

Le contraste entre les banquets publics, où règne la confusion, et les «conviti famigliari » («festins familiers »), moments bénis de bienveillance et de paix, illustre bien l'idée guazzienne de la «civile conversation »dans sa variante « domestique », qui raccourcit la distance entre l'homme extérieur et l'homme intérieur $^{19}$.

Luc Vaillancourt (2005: 84), qui a analysé les liens entre la Civil conversatione et le genre familier tel qu'il a été défini par Érasme, note :

Le sermo, tel que conçu par Érasme et exploité par Guazzo, constitue en quelque sorte une porte d'entrée de l'individualisme en littérature parce qu'il autorise, voire encourage, l'expression de soi, alors que le mode polémique ne la cautionne que si elle peut servir

${ }^{18}$ Sur la conception humaniste du mariage comme une forme supérieure et privilégiée de l'amitié cf. Furey (2011).

${ }^{19}$ Cf. aussi les observations de Amyrose McCue Gill (2011) sur l'usage du mot « compagna » dans les réflexions albertiennes sur l'amitié entre les époux : "Originating from the Latin cum panis, the Italian compagno/a means "con pane", or "one with whom bread is shared". Thus are companionship and friendship first linked by I libri [della famiglia]: in the Nicomachean Ethics, Aristotle emphasizes the importance of eating together - sharing salt - as a means of coming to perfect friendship [...]». 
l'argumentation. Certes, il n'est pas évident de faire le départ entre ce qui relève d'une stratégie argumentative et ce qui correspond à une réalité extratextuelle (la chose est-elle même possible ?), mais la prédilection dont jouit le mode conversationnel à l'époque classique témoigne à tout le moins d'un désir de se démarquer du conformisme sociodiscursif qui déterminait jusqu'alors non seulement les discours, mais les identités mêmes.

Face à la complexité des codes qui caractérise d'autres types de communication décrits dans la Civil conversatione, la «marital conversazione », telle que Stefano Guazzo la dépeint, est un modèle de communication le plus proche de l'idéal de la transparence de la parole. 


\section{BIBLIOGRAPHIE}

Alberti, Leon Battista (1994). I libri della famiglia, R. Romano \& A. Tenenti \& F. Furlan (eds.). Torino: Einaudi.

ArISTOTE (2014). Éthique à Nicomaque (VIII, 1158b). <http://www.echosdumaquis.com/Accueil/ Textes_(A-Z)_files/E\%CC\%81thique\%20a\%CC\%80\%20Nicomaque.pdf >.

Bernardino da Siena (1989). Prediche Volgari sul Campo di Siena 1427. Vol. II. Éd. Carlo Delcorno. Milano : Rusconi.

BoccazzI, Gaudenzio (1991). La civil conversatione : Montaigne e Stefano Guazzo. In Enea Balmas (éd.), Montaigne e l'Italia (pp. 295-303). Moncalieri-Genève : Slatkine.

Casagrande, Carla (1991). La femme gardée. In Georges Duby \& Michelle Perrot (dir.), Histoire des femmes en Occident. T. 2. Christiane Klapisch-Zuber (dir.), Le Moyen Âge (pp. 83-116). Paris : Plon.

Courtine, JeAn-JACQues \& Haroche, Claudine (1994). Histoire du visage. Exprimer et taire ses émotions : XVI - début XIX ${ }^{e}$ siècle. Paris : Payot.

De Matteis, Maria Consiglia (1982). Idee sulla donna nel Medioevo : fonti e aspetti giuridici, antropologici, religiosi, sociali e letterari della condizione femminile. Bologna : Patron.

Della Casa, Giovanni (1666). Galatée, ou L'art de plaire dans la conversation, Duhamel, J.-B. (trad.). Paris : René Guignard.

- (1994). Galateo. Éd. Stefano Prandi. Torino : Einaudi.

Dolce, Lodovico (1545), Dialogo [...] della institution delle donne. Secondo li tre stati, che cadono nella vita humana. Venezia : Gabriel Giolito. <http://www.bibliotecaitaliana.it/indice/ visualizza_testo_html/bibit000361>.

Duby, Georges (1981). Le chevalier, la femme et le prêtre. Le mariage dans la France féodale. Paris : Hachette.

Frigo, DANIEla (1990). Civil conversatione e pratica del mondo: le relazioni domestiche. In Giorgio Patrizi (éd.), Stefano Guazzo e la civil conversazione (pp. 121-145). Roma : Bulzoni.

Fubini LeUZZI, MARIA (1996). Vita coniugale e vita familiare nei trattati italiani fra XVI e XVII secolo. In Gabriella Zarri (éd.), Donna, disciplina, creanza cristiana. Roma : Edizioni di Storia e Letteratura.

FumAROLI, MARC (1994). Trois institutions littéraires. Paris : Gallimard.

Furey, Constance M. (2011). Bound by Likeness : Marital Friendship in Early Modern Humanism. In Daniel T. Lochman, Maritere López \& Lorna Hutson, Discourses and Representations of Friendship in Early Modern Europe, Burlington : Ashgate Press.

Furlan, Francesco (2004). La donna, la famiglia, l'amore tra medioevo e rinascimento. Firenze : Olschki.

Guazzo, Stefano (1580). La civile conversation : divisée en quatre livres. Trad. Gabriel Chappuys. Lyon : Jean Beraud.

- (1993). La civil conversazione. Éd. Amadeo Quondam. Modena : Panini.

LEUSHUIS, REINIER (2003). Le Mariage et «l'amitié courtoise » dans le dialogue et le récit bref de la Renaissance. Firenze : Olschki.

Loba, Anna (2010). Enseignement de Prudence : réflexions sur le mariage de Christine de Pizan à Érasme. Romanica Cracoviensia. Numéro spécial : Marcela Świątkowska (éd.), Fontes Christiance aux $X V^{e}$ et $X V I^{e}$ siècles : lectures, inspirations, contestations, 47-56.

LOJACONO, ETTORE (2004). Socrate e l'honnete homme nella cultura dell'autunno del Rinascimento francese e in René Descartes. In Ettore Lojacono (éd.), Socrate in Occidente (pp. 103-146). Firenze : Le Monnier.

Magli, PAtRizia (1995). Il volto e l'anima. Fisiognomica e passioni. Milano : Bompiani. 
Majorana, Bernadette (1996). Finzioni, imitazioni, azioni : donne e teatro. In Gabriella Zarri (éd.), Donna, disciplina e creanza cristiana dal XV al XVII secolo (pp. 121-139). Roma: Edizioni di Storia e Letteratura.

McCue Gill, Amyrose (2011). Rereading I libri della famiglia : Leon Battista Alberti on Marriage, Amicizia and Conjugal Friendship. California Italian Studies Journal, 2, Issue 2. <https://escholarship.org/uc/item/9t3049v8\#page-1>.

MuZZarelli, Maria GuisepPIna (2008). Guardaroba medievale. Vesti e società dal XIII al XVI secolo. Bologna : Il Mulino.

PANiChI, Nicola (2004). I vincoli del disinganno. Per una nuova interpretazione di Montaigne. Firenze : Olschki.

PAtrizi, Giorgio (1990). Una retorica del molteplice. Forme di vita e forme del sapere nella Civil conversatione. In Giorgio Patrizi (éd.), Stefano Guazzo e la civil conversazione (pp. 47-94). Roma : Bulzoni.

QuONDAM, AmAdEO (1993). Introduzione. In Guazzo, S. La civil conversazione (pp. XXIII-LX). Modena : Panini.

REMOTTI, FRANCESCO (1993). Luoghi e corpi : antropologia dello spazio, del tempo e del potere. Torino : Bollati Boringhieri.

RonCHI, RocCO (2003). Teoria critica della comunicazione. Milano : Mondadori.

SNYDER, JON R. (1995). Appunti sulla politica e l'estetica della dissimulazione tra Cinque e Seicento. Cheiron, 22, 23-43.

VAILlANCOURT, LuC (2005). Topologie du registre conversationnel chez Érasme et Guazzo. Tangence, 79, Automne, 75-85. <http://www.erudit.org/revue/tce/2005/v/n79 /012852ar.html?vue $=$ integral>.

VANNI, LAURA (2006). La cura del corpo nei galatei del rinascimento. In Amadeo Quondam (éd.), Giovanni Della Casa. Un Seminario per il centenario (pp. 383-390). Roma : Bulzoni.

Vecchio, Silvana (1991). La bonne épouse. In Georges Duby \& Michelle Perrot (dir.), Histoire des femmes en Occident. T. 2. Christiane Klapisch-Zuber (dir.), Le Moyen Âge (pp. 117-145). Paris : Plon.

ZANCAN, MARINA (1983). La donna e il cerchio nel Cortegiano di B. Castiglione. Le funzioni del femminile nell'immagine di corte. In Marina Zancan (éd.), Nel cerchio della Luna. Figure di donna in alcuni testi del XVI secolo (pp. 13-56). Venezia : Marsilio. 